\title{
Researching on General Modeling Method for a Class of Spoke Inclined Parallel Mechanism
}

\author{
Wei Liu*, Longying Zhu and Zebing Xu
}

School of Automotive Engineering, Yancheng Institute of Technology, Yancheng, 224051, China

\begin{abstract}
With the deepening of researching on parallel mechanism, a variety of parallel mechanism and corresponding modeling method are presented, but these methods are not universal. So this paper deals with a class of spoke inclined parallel mechanism as the object, trying to get the general modeling method of kinematics and dynamics, which will provide convenient on this kind of parallel mechanism. Kinematics model with inverse selection criteria is established using geometric method, dynamics model is established by Lagrange method, then the mapping relationship between inputoutput displacement and driving force of the parallel mechanism are presented; Set $45^{\circ}$ angle as an example, the prototype of spoke inclined parallel mechanism is developed based on dSPACE real-time simulation system, the validation experiments and rotation performance test experiments are done using the methods combining the theoretical modeling and experimental validation. The experimental results show that the mathematical model is correct, the parallel mechanism has the advantage of low inertia and large angle, the maximum angle of the prototype can reach $51.4^{\circ}$. The parallel mechanism in this paper can be used for automobile coating and manufacturing, and the research will lay the foundation for further application in mechanical industry.
\end{abstract}

Keywords: Dynamics, general modeling method, kinematics, parallel mechanism, prototype.

\section{INTRODUCTION}

Along with the development of society and the improvement of the automobile industry, parallel mechanism has become one of the research focuses [1]. 6-DOF (degree of freedom) parallel mechanism has widely applied in motion simulation, machine tools, medical device and other fields for its high stiffness, fast response and small error accumulation [2]. The research on kinematics and dynamics of 6-DOF parallel mechanism is a necessary condition to its application in mechanical industry. An algebraic elimination method is presented by Zhang [3] to solve the forward kinematics of parallel mechanisms, and can get all solutions of original equations without extraneous roots. Han [4] proposed a fast forward algorithm solution of parallel mechanism; and computation time is reduced to $50 \%$ using iteration method in the premise of the same calculation accuracy. Dynamic model of a kind of 5-DOF parallel mechanism is established by Chen [5] using the principle of virtual work and the model is verified by simulation using ADAMS. To improve the security of the wind tunnel test, kinematics of a 6PPS parallel mechanism is analyzed, and the dynamic characteristics are summarized by Sun [6]. Based on genetic algorithm, the driving force distribution of the six pyramid type parallel mechanism in the redundant tasks is optimized, and the method for obtaining the distribution of minimum energy consumption by Liu [7].
Since the advent of Stewart parallel mechanism, parallel mechanisms with different driving source, connection mode and setting of the structure have been emerged in an endless stream [8]. Throughout the existed research on kinematics [9] and dynamics [10] of parallel mechanisms, most models are established according to the analysis of a specific configuration; the general modeling methods for parallel mechanisms are relatively rare. In this paper, a spoke inclined parallel mechanism has been brought out. The general modeling method of kinematics and dynamics are studied which will lay a theoretical foundation for the application of this type of parallel mechanisms in mechanical industry.

\section{SPOKE INCLINED PARALLEL MECHANISM}

\subsection{Structure and Working Principle}

The structure diagram of spoke inclined parallel mechanism is presented as shown in Fig. (1).

The spoke inclined parallel mechanism consists of load platform (1), Hooke joint (2), connecting rod (3); spherical joint (4), lead rail (5) and sliding pair (6). The system has defined two coordinate systems: one for the moving coordinate system $O^{\prime}-x^{\prime} y^{\prime} z^{\prime}$, which is fixedly connected with the center of load platform; another is the inertial coordinate system $O-x y z$, which origin is in the intersection of the extend line of the lead rails. The six lead rails $\operatorname{DiD}^{\prime} i(i=1-6)$ are inclined to the ground with the angle $\theta$ from 0 to 90 degrees, and the performance of the spoke inclined parallel mechanism varies when the angle $\theta$ changes. The lower end of 


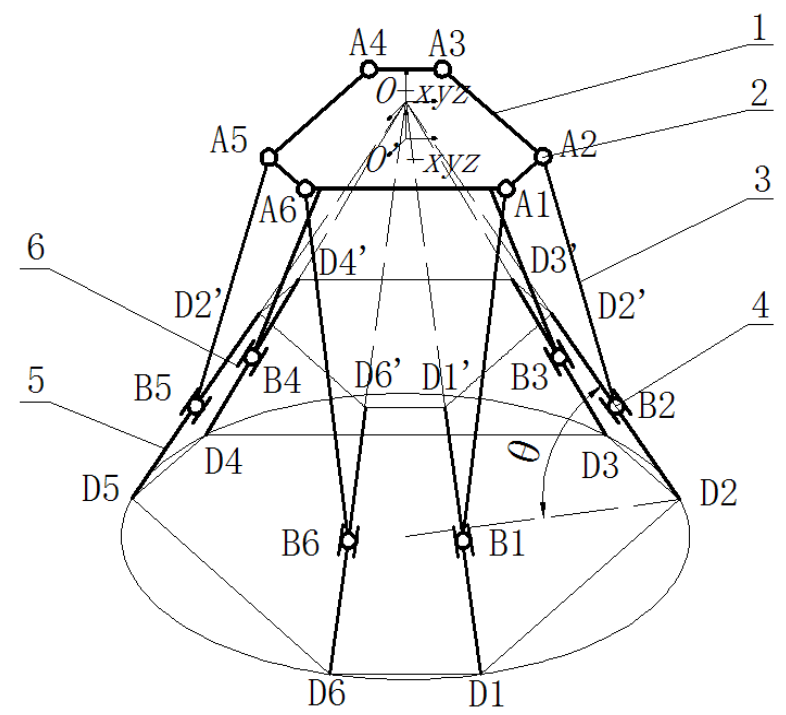

Fig. (1). Sketch map of spoke inclined parallel mechanism.

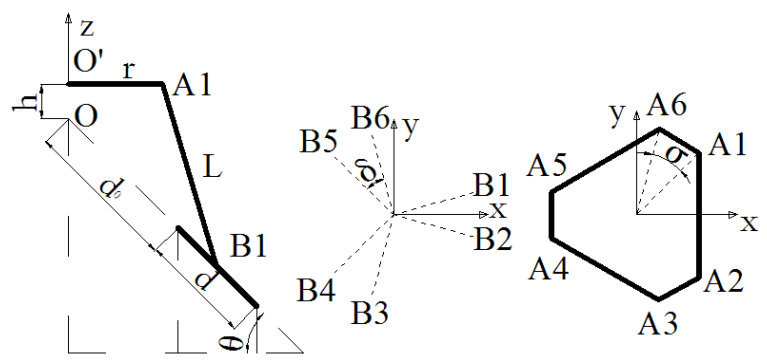

Fig. (2). Projection view of spoke inclined parallel mechanism.

connecting rod connects with the motor rotor through a spherical joint at $B i(i=1-6)$, and the upper end of connecting rod connects with load platform through a Hooke joint at $\mathrm{Ai}$ $(i=1-6)$. The six motor rotors move back and forth in the corresponding lead rail, which will lead the six freedom space movement of the load platform.

\subsection{Calculation of the Degree of Freedom}

The degree of freedom of spoke inclined parallel mechanism is calculated according to the Grubler formula:

$$
f_{0}=6 m-\sum_{i=1}^{n} f_{i}
$$

In the formula, $f_{0}$ is the degree of freedom of the spoke inclined parallel mechanism; $m$ is the number of the moving parts; $n$ is the number of the kinematic pairs; $f_{i}$ is the product of the number of restricted freedom of kinematic pairs and the number of the kinematic pairs.

The moving parts of spoke inclined parallel mechanism consist of six motor rotors, six connecting rods and a load platform, which total number is 13 . The kinematic pairs of spoke inclined parallel mechanism consist of six sliding pair, six Hooke joints and six spherical joints, which total number is 18 . The number of restricted freedom of spherical joint is 3 ; the number of restricted freedom of Hooke joint is 4 and the number of restricted freedom of sliding pair is 5 . Then the degree of freedom of spoke inclined parallel mechanism can be calculated:

$f_{0}=6 \times 13-6 \times(5+4+3)=6$

It is thus clear that the degree of freedom is 6 ; the mechanism can achieve the linear motion along the three coordinate axes and the rotary motion around the three coordinate axes.

\section{THE GENERAL MODELING METHOD}

\subsection{Kinematic Modeling}

The spoke inclined parallel mechanism is a complex system with multi input and multi output. The parameters of the mechanism are defined from three projection view as shown in Fig. (2) before the establishment of mathematical model.

In Fig. (2), $r$ is the circumradius of the load platform; $h$ is the distance between the two coordinate origins; $d$ is the range of lead rail; $d_{0}$ is the distance between lead rail and the coordinate origin $O ; \delta$ is the angle of the projection of lead rails; $\sigma$ is the angle of the joints of load platform.

If the center of the load platform is given as $\boldsymbol{P}=\left[x_{p}, y_{p}, z_{p}, \alpha, \beta, \gamma\right]^{T}$, the coordinate of the joint $A i$ in $O^{\prime}-x^{\prime} y^{\prime} z^{\prime}$ can be expressed as: 


$$
a i=[r \cos \sigma, r \sin \sigma, 0]^{T}
$$

Then the coordinate of the joint $A i$ in $O-x y z$ can be expressed according to RPY description:

$$
\left[\begin{array}{c}
A i_{x} \\
A i_{y} \\
A i_{z}
\end{array}\right]=\left[\begin{array}{c}
x_{p} \\
y_{p} \\
z_{p}+h
\end{array}\right]+\boldsymbol{R}\left[\begin{array}{c}
a i_{x} \\
a i_{y} \\
a i_{z}
\end{array}\right]
$$

And $\boldsymbol{R}$ is the institutional transformation matrix.

The distance between motor rotors and $D i$ is defined as $d_{i}$, so the absolute coordinate of motor rotors can be expressed as $B i$ :

$$
\left[\begin{array}{l}
B i_{x} \\
B i_{y} \\
B i_{z}
\end{array}\right]=\left[\begin{array}{l}
\left(d_{0}+d_{i}\right) \cos \theta \sin (\delta / 2) \\
\left(d_{0}+d_{i}\right) \cos \theta \cos (\delta / 2) \\
\left(d_{0}+d_{i}\right) \sin \theta
\end{array}\right]
$$

According to the geometric conditions of the mechanism, the distance between Hooke joint of load platform $A i$ and corresponding motor rotor $B i$ is always the constant $L$. Then the kinematic equations are available for:

$$
\left(A i_{x}-B i_{x}\right)^{2}+\left(A i_{y}-B i_{y}\right)^{2}+\left(A i_{z}-B i_{z}\right)^{2}=L^{2}
$$

Combined with formula (4-6), the coordinate of the six motor rotors can be calculated, and the relationship between input and output displacement of the mechanism is established. Note that formula (6) is one basic quadratic equation, so there are two groups of real solutions, and the solution between 0 and $\left|D i D i^{\prime}\right|$ is the efficient solution of kinematic equations.

\subsection{Dynamic Modeling}

There are different dynamic modeling methods such as Newton Euler method, Lagrange method and the Kaine equation, and these methods are equivalent in essence. Lagrange method can obtain the dynamic equations from the view of energy, and the dynamic characteristics of the mechanism can be realized. Thus, Lagrange method is more popular for its convenience and practicality.

Lagrange function $\boldsymbol{\Gamma}$ is defined as the difference between kinetic energy $\boldsymbol{K} \boldsymbol{E}$ and potential energy $\boldsymbol{K P}$ of the system. For spoke inclined parallel mechanism, the numerical value of Coriolis force and centrifugal force is too small, which can be neglected. Then, Lagrange equation can be written in vector form:

$$
\frac{d}{d t}\left(\frac{\partial}{\partial \dot{\boldsymbol{P}}}\right)-\frac{\partial}{\partial \boldsymbol{P}}=
$$

In the formula, $\boldsymbol{P}$ is the pose of load platform; $\boldsymbol{\tau}$ is the driving force vector of the motors. If the kinetic energy and potential energy are expressed as follows:

$$
\left\{\begin{array}{l}
\boldsymbol{K} \boldsymbol{E}=\frac{1}{2} \dot{\boldsymbol{P}}^{T} \boldsymbol{M}(\boldsymbol{P}) \dot{\boldsymbol{P}} \\
\boldsymbol{K P}=F(\boldsymbol{P})
\end{array}\right.
$$

Then, the standard form of Lagrange equations can be obtained as follows:

$$
M(P) \ddot{P}+G(P)=\tau
$$

In the formula, $\boldsymbol{M ( P )}$ is the inertial matrix, which can be obtained by the kinetic energy of the system. It consists of the following two parts: one part is the inertia matrix of load platform; the other part is the inertia matrix of connecting $\operatorname{rod} . \boldsymbol{G}(\boldsymbol{P})$ is the partial derivatives of the potential energy of the system.

The kinetic energy of the spoke inclined parallel mechanism consists of kinetic energy of the load platform and kinetic energy of the connecting rod. Define $m$ as the mass of load platform; $\left[\mathbf{V} \boldsymbol{p}, \boldsymbol{\omega}_{\boldsymbol{p}}\right]^{\mathrm{T}}$ as systemic velocity; $\boldsymbol{I}$ as the moment of inertia around the axis in absolute coordinate, then the kinetic energy of the system can be expressed as follows:

$$
\boldsymbol{K} \boldsymbol{E}_{1}=\frac{1}{2}\left[m \boldsymbol{V}_{p}^{2}+\boldsymbol{\omega}_{p}^{T} \boldsymbol{R} \boldsymbol{\boldsymbol { R } ^ { T }} \boldsymbol{\omega}_{\boldsymbol{p}}\right]
$$

By above knowable, the six motors are fixed to the bottom plane, it can greatly reduced the moment of inertia of the moving parts. Regarding the connecting rod as particles, the calculation of the kinetic energy of $i$ can be expressed as:

$\boldsymbol{K} \boldsymbol{E}_{2}=\frac{1}{2} m_{l} \sum_{i=1}^{6}\left(\boldsymbol{V}_{A i}+\boldsymbol{V}_{B i}\right)^{T}\left(\boldsymbol{V}_{A i}+\boldsymbol{V}_{\boldsymbol{B}}\right)$

In the formula, $m_{l}$ is the mass of connecting rod. To get the inertia matrix of the connecting rod, it is necessary to express $\boldsymbol{V}_{\boldsymbol{A} i}$ and $\boldsymbol{V}_{\boldsymbol{B} \boldsymbol{i}}$ using the derivative of $\boldsymbol{P}$. The Jacobian matrix $\boldsymbol{J}$ of the spoke inclined parallel mechanism conveys the relationship between input speed and output speed of the system. So $\boldsymbol{V}_{\boldsymbol{B}}$ can be expressed as:

$\boldsymbol{V}_{\boldsymbol{B}}=-\left(\frac{\partial F}{\partial \boldsymbol{B}}\right)^{-1} \cdot \frac{\partial F}{\partial \boldsymbol{P}} \cdot \dot{\boldsymbol{P}}=[\boldsymbol{J}] \cdot \dot{\boldsymbol{P}}$

As $\boldsymbol{V}_{A i}$ and $\boldsymbol{P}$ are in the same rigid body, So $\boldsymbol{V}_{A}$ can be expressed according to the vector $\boldsymbol{r}_{A i}$ which from center of the load platform to each Hooke joint:

$\boldsymbol{V}_{A}=\boldsymbol{V}_{\boldsymbol{P}}+\omega_{\boldsymbol{P}} \times \boldsymbol{r}_{A i}=[\boldsymbol{G}] \dot{\boldsymbol{P}}$

Then, the inertia matrix of connecting rod can be obtained as $\boldsymbol{M}_{2}$ :

$\boldsymbol{M}_{2}=\sum_{i=1}^{6}([\boldsymbol{G}]+[\boldsymbol{J}])^{T}([\boldsymbol{G}]+[\boldsymbol{J}])$

Potential energy of load platform is determined by the distance between center of mass and the origin of the coordinate $O-x y z$. Regarding the connecting rod as particles, $\boldsymbol{K P}$ is only related to the coordinate of the center of mass. Then the expression of $\boldsymbol{K} \boldsymbol{P}$ can be written as:

$$
\boldsymbol{K} \boldsymbol{P}=m g\left(z_{p}+\cos \beta \cos \alpha\right)+\sum_{i=1}^{6} \frac{m_{l i} g}{2}\left(z_{A i}+z_{B i}\right)
$$

To complete a six dimensional trajectory task, the driving force of the motors $\tau$ can be calculated based on formula (9$15)$ :

$$
\tau=\left[\boldsymbol{M}_{1}+\boldsymbol{M}_{2}\right] \ddot{\boldsymbol{P}}+\frac{\partial \boldsymbol{K} \boldsymbol{P}}{\partial \boldsymbol{P}}
$$




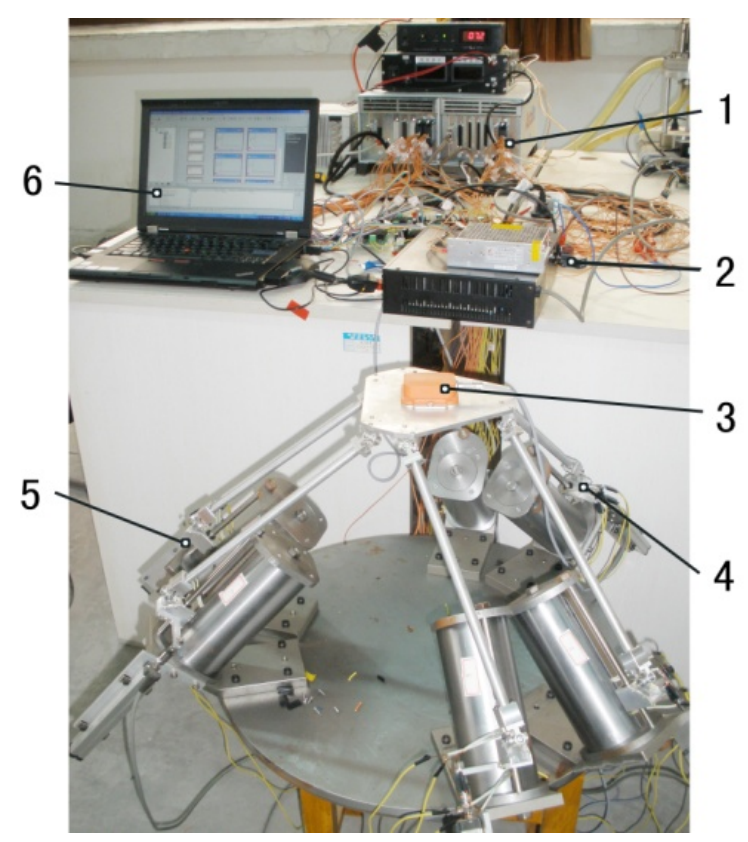

Fig. (3). Prototype of spoke inclined parallel mechanism.

Formula (16) reflects the mapping relationship between the input force and output displacement of the spoke inclined parallel mechanism. With different the angle $\theta$, the mechanism will have different structure and performance characteristics. And we can analyze kinematic and dynamic performance of this class of spoke inclined parallel mechanism through this general mathematical model.

\section{PROTOTYPE AND EXPERIMENT}

\subsection{Prototype and its Control System}

For further research on spoke inclined parallel mechanism and its performance, it is necessary to develop the prototype of this mechanism. Driven by linear motors, the prototype has been developed with the angle $\theta=45^{\circ}$ which is shown in Fig. (3).

The control system consists of dSPACE real time simulation system (1), current sensor (2), pose sensor (3), linear motor (4), displacement sensor (5) and upper monitor (6). Notice that the force of linear motor is not easy to be measured directly, so the current is measured to calculate the driving force according to the formula of Ampere force:

$$
F=B I L
$$

When we input a six dimensional trajectory task to the upper monitor, the displacement and force of the linear motor can be calculated based on mathematical model. Orders are provided from dSPACE to linear motors, and corresponding signals are measured by current sensors and displacement sensors. Based on the feedback of force and displacement signals to dSPACE, closed-loop control is realized to improve the accuracy of the motion of load platform according to target trajectory. At the same time, displacement along the three axes and the rotation around the three axes are measured by pose sensor, the signals are delivered to upper monitor for data recording and real-time monitoring.

\subsection{Validation Experiments}

The correctness of the kinematic and dynamic model of the spoke inclined parallel mechanism is the foundation for the next step of research. So validation experiments are done according to the six dimensional trajectory tasks as follows:

$$
\boldsymbol{P}=\left(\begin{array}{l}
x_{p} \\
y_{p} \\
z_{p} \\
\alpha \\
\beta \\
\gamma
\end{array}\right)=\left(\begin{array}{l}
5 \sin (t) \\
5 \sin (2 t) \\
3 \sin (t) \\
0.0436 \sin (t) \\
0.0436 \sin (2 t) \\
0.0872 \sin (t)
\end{array}\right)
$$

Based on the kinematic model, the displacement variation of the six linear motors can be calculated using Matlab, which is shown in Fig. (4).

According to the dynamic model, the driving force variation of the six linear motors can be calculated using Matlab, which is shown in Fig. (5).

When the direction of the driving force is from the bottom to the top, the value of the driving force is considered to be positive; when the direction of the driving force is from the top to the bottom, the value of the driving force is considered to be negative. Then, the variation of current can be calculated according to formula (17). Based on the signal of displacement and current, the spoke inclined parallel mechanism is controlled by dSPACE. At the same time, the signals of displacement along the three axes and rotation around the three axes are recorded by the pose sensor. To verify the 


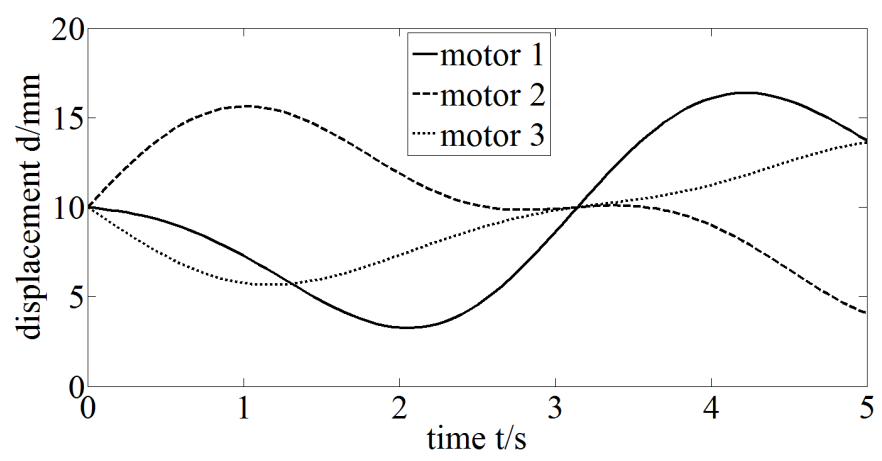

a. displacement of motor 1-3

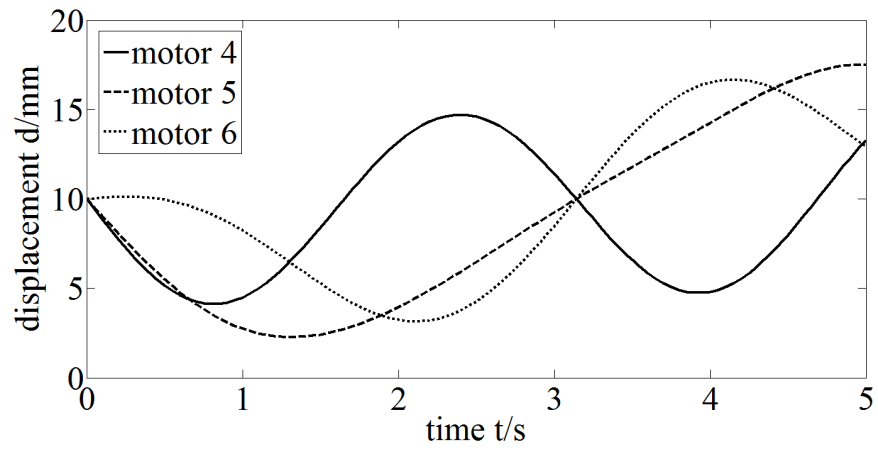

b. displacement of motor 4-6

Fig. (4) Displacement variation of the motors.

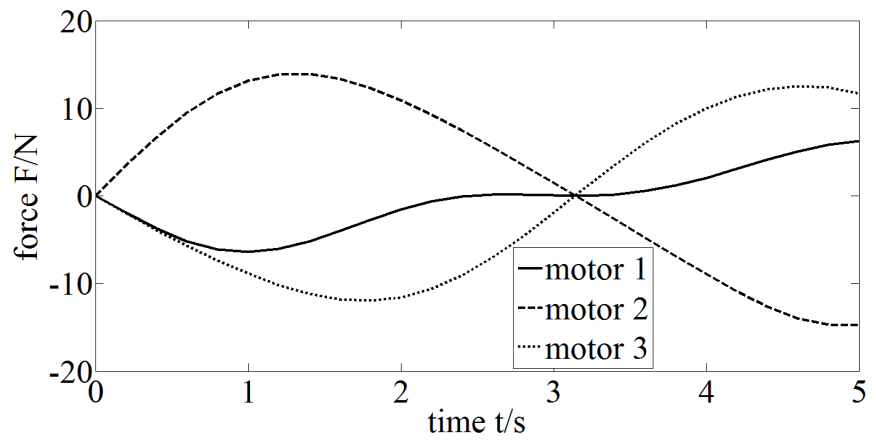

a. force of motor 1-3

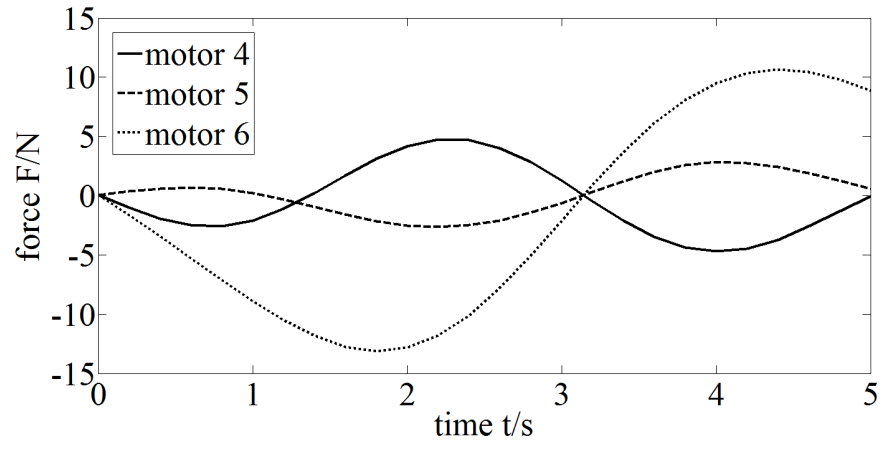

b. force of motor 4-6

Fig. (5). Force variation of the motors. 


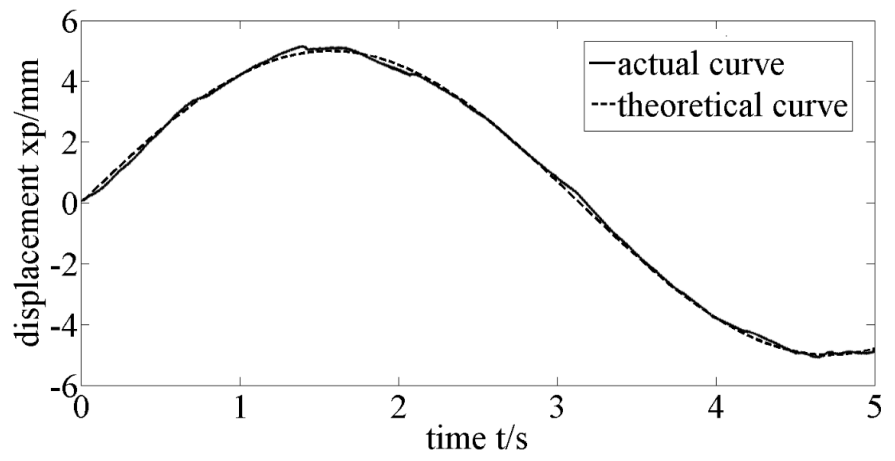

a. Displacement along the $x$ axis

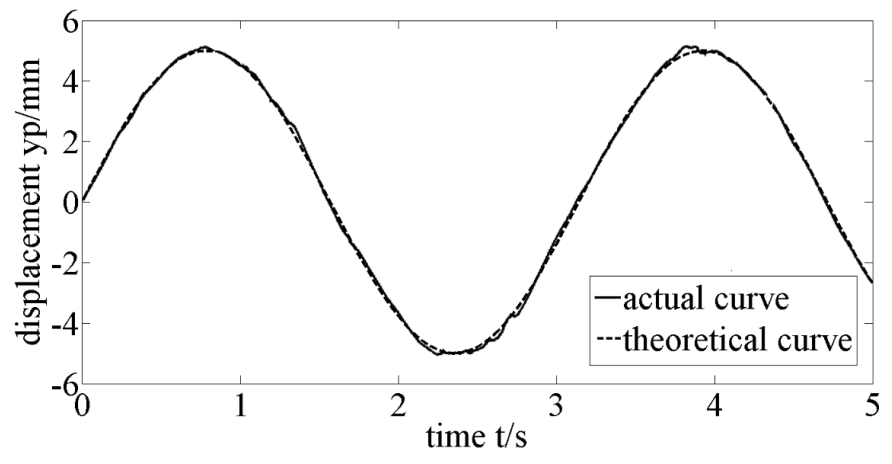

b. Displacement along the $y$ axis

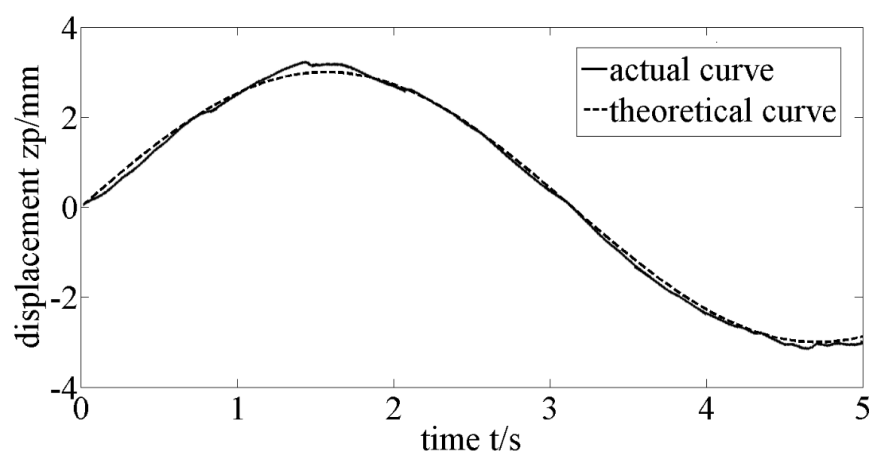

c. Displacement along the $z$ axis

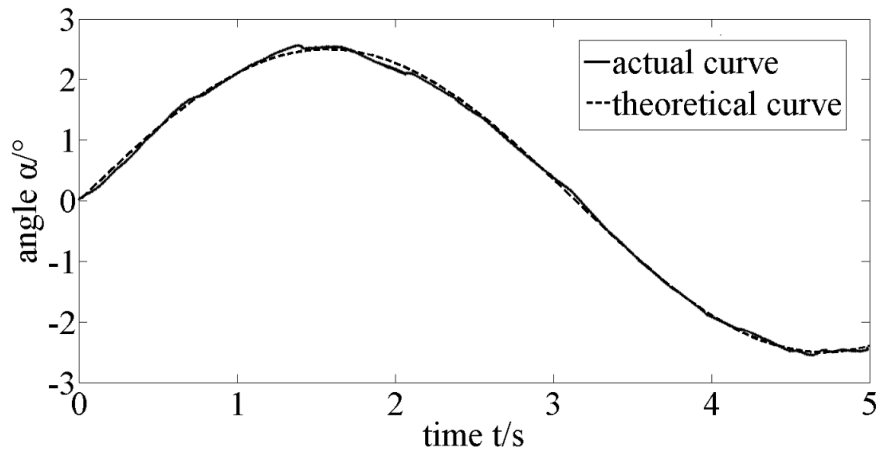

d. angle around the $x$ axis 


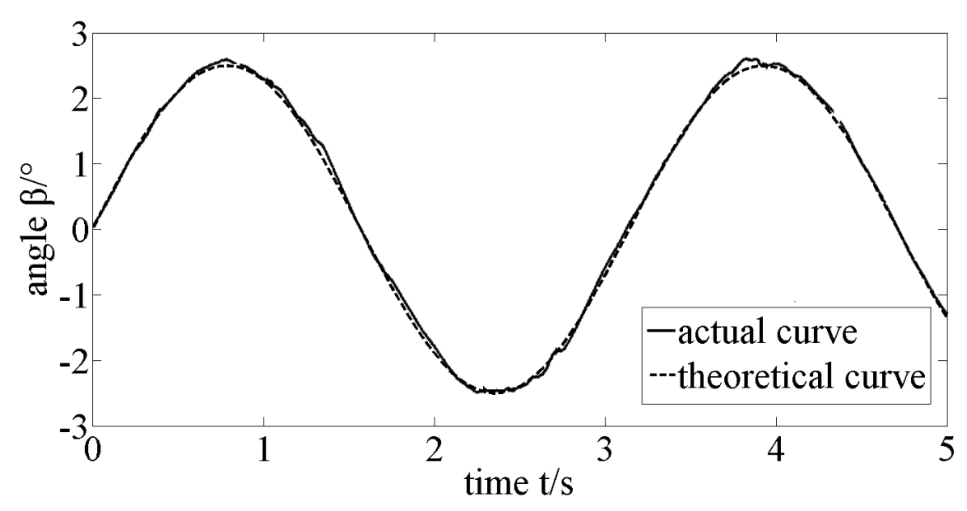

e. angle around the $y$ axis



f. angle around the $z$ axis

Fig. (6). Comparison between theoretical and the actual variation of the load platform.

correctness of the model, the theoretical curves and the actual curves are plotted in one figure, as shown in Fig. (6).

From the figure we can see that the variation of the theoretical curves and the actual curves are comparatively unanimous. But there are still have some error, and the cause of the errors exist mainly in the following two aspects: one for the manufacturing error in the process of prototype production. Though the spoke inclined parallel mechanism can reduce the error accumulation of branched chains, but there still have a certain gap between theoretical size and the actual size of the spare parts. This kind of error can be corrected by modified the model according to the actual size of the prototype. Another reason for the error is sensor error. This kind of error can be divided into installation error and measurement error, which can be reduced by improving the accuracy of manufacturing of the brackets or using the sensors with high performance. The results of validation experiments show that the kinematic and dynamic model of the spoke inclined parallel mechanism is correct.

\subsection{Rotation Performance Test Experiment}

In order to know the limit performance of the spoke inclined parallel mechanism, rotation performance test experiment is done to reveal the maximum rotation angle of the mechanism through the boundary condition method. The situation that the mechanism has a maximum rotation angle happens when the motor rotor which is farthest from the coordinate axis goes to the travel limit. And it can be considered to be the maximum rotation angle around the certain axis.

Specific steps of the rotation performance test experiment are as follows: first, the coordinate of boundary points of the load platform are calculated using the mathematical model. Second, a trajectory of load platform is fitted including these boundary points. Then, when the mechanism is working under the target trajectory, the data of the rotation angle are recorded by the pose sensor. Finally, by repeating the experiment, the maximum angle of load platform around the three axes is obtained as shown in Table $\mathbf{1}$.

The table shows that the maximum angle of the spoke inclined parallel mechanism which around the $z$ axis is $51.4^{\circ}$, and the maximum angle happens near the location $(0,0,0)^{\mathrm{T}}$. The results of rotation performance test experiments show that the spoke inclined parallel mechanism has good performance in rotation.

Table 1. Maximum rotation angle of parallel mechanism.

\begin{tabular}{|c|c|}
\hline axis & Maximum angle $/{ }^{\circ}$ \\
\hline \hline$x$ & $41.1^{\circ}$ \\
\hline$y$ & $41.5^{\circ}$ \\
\hline$z$ & $51.4^{\circ}$ \\
\hline
\end{tabular}




\section{CONCLUSION}

The paper presents a kind of parallel mechanism, which can be used in automobile coating and manufacturing, there are three conclusions:

(1) The general mathematics model of kinematics and dynamics are established for a class of spokes inclined parallel mechanism using geometric method and Lagrange method, which can obtain the mapping relationship between the input and output of displacement and driving forces.

(2) A prototype is produced with the angle $\theta=45^{\circ}$, which is controlled by dSPACE real-time simulation system. The validation experiments show that the kinematic and dynamic model of the spoke inclined parallel mechanism is correct.

(3) The rotation performance test experiments are done to reveal the maximum rotation angle of the mechanism. The results show that the spoke inclined parallel mechanism has good performance in rotation, and the maximum rotation angle around $z$ axis can reach $51.4^{\circ}$. The research will lay a good foundation for the applications in mechanical industry.

\section{CONFLICT OF INTEREST}

The author confirms that this article content has no conflict of interest.

\section{ACKNOWLEDGEMENTS}

At the point of finishing this paper, I'd like to express my sincere thanks to Dr. Zhu who has given me so much useful advices on my writing, and has tried her best to improve my paper.

The paper is supported by:
(1) "National Natural Science Foundation of China (51405419)".

(2) "Cooperative Innovation Found project of Jiangsu Province (BY2014108-17)".

\section{REFERENCES}

[1] W. Liu and H. Zhao, "Application of improved particle swarm optimization for forward positional analysis of parallel mechanism", Machinery Design and Manufacture, vol. 2, pp. 181-183, February 2014.

[2] F. Liu, X. Zhang and Y. Wei, "The forward kinematics analysis of 6-UPS parallel mechanism based on newton iteration", Machinery Design and Manufacture, vol. 5, pp. 173-174, May 2014.

[3] Y. Zhang, Q. Liao and S. Wei, "Forward displacement analysis of a general 6-4 in-parallel platform", Journal of Mechanical Engineering, vol. 48, pp. 26-27, May 2012.

[4] F. Han, D. Zhao and T. Li, "A fast forward algorithm for 3-RPS parallel mechanism", Transactions of the Chinese Society of Agricultural Machinery, vol. 42, pp. 229-225, April 2011.

[5] X. Chen, W. Feng and Y. Zhao, "Dynamics model of 5-dof parallel robot mechanism", Transactions of the Chinese Society of Agricultural Machinery, vol. 44, pp. 236-238, January 2013.

[6] X. Sun, Z. Xie and K. Lin, "Dynamics analysis and simulation of 6-PSS flexible parallel robot", Transactions of the Chinese Society of Agricultural Machinery, vol. 43, pp. 194-197, June 2012.

[7] W. Liu and S. Chang, "Drive optimization of parallel robot under redundant tasks based on genetic algorithm", Transactions of the Chinese Society of Agricultural Machinery, vol. 43, pp. 221-225, April 2012.

[8] M. Stone, R. Benneweis and J Van, "Evolution of electronics for mobile agricultural equipment", Transactions of the ASABE, vol. 51, pp. 385-386, May 2008.

[9] T. Grift, Q. Zhang, and N. Kondo, "A review of automation and robotics for the bio industry", Journal of Bio Mechatronics Engineering, vol. 1, pp. 37-38, January 2008.

[10] X. Tan and Z. Xie, "Study on flexible dynamics of 6-PUS parallel support robot", Machinery Design and Manufacture, vol. 2, pp. 133-133, February 2014.

Received: November 21, 2014

(C) Liu et al.; Licensee Bentham Open.

This is an open access article licensed under the terms of the Creative Commons Attribution Non-Commercial License (http://creativecommons.org/licenses/by-nc/3.0/) which permits unrestricted, non-commercial use, distribution and reproduction in any medium, provided the work is properly cited. 